\title{
Direct Imaging of Drug Distribution and Target Engagement of the PARP Inhibitor Rucaparib
}

\author{
Susanne Kossatz ${ }^{1}$, Brandon Carney ${ }^{1,2}$, Christopher Farley ${ }^{2}$, Wolfgang A. Weber ${ }^{1,3,4}$, Charles M. Drain², \\ and Thomas Reiner ${ }^{1,3}$ \\ ${ }^{I}$ Department of Radiology, Memorial Sloan Kettering Cancer Center, New York, New York; ${ }^{2}$ Hunter College and Graduate Center of the \\ City University of New York, New York, New York; ${ }^{3}$ Department of Radiology, Weill Cornell Medical College, New York, New York; and \\ ${ }^{4}$ Department of Nuclear Medicine, Technical University Munich, Munich, Germany
}

\begin{abstract}
Poly(ADP-ribose)polymerase (PARP) inhibitors have emerged as potent antitumor drugs. Here, we describe the intrinsic fluorescence properties of the clinically approved PARP inhibitor rucaparib and its potential to directly measure drug distribution and target engagement-a critical factor for understanding drug action and improving efficacy. Methods: We characterized the photophysical properties of rucaparib and determined its quantum yield and lifetime. Using confocal microscopy and flow cytometry, we imaged the intracellular distribution of rucaparib and measured uptake and release kinetics. Results: Rucaparib has an excitation/emission maximum of $355 / 480 \mathrm{~nm}$ and a quantum yield of 0.3 . In vitro timelapse imaging showed accumulation in cell nuclei within seconds of administration. Nuclear rucaparib uptake increased with higher PARP1 expression, and we determined an intracellular half-life of 6.4 h. Conclusion: The label-free, intrinsic fluorescence of rucaparib can be exploited to interrogate drug distribution and target binding, critical factors toward improving treatment efficacy and outcome.
\end{abstract}

Key Words: PARP1; rucaparib; fluorescence imaging; indole chromophore; drug-target interactions

J Nucl Med 2018; 59:1316-1320

DOI: 10.2967/jnumed.117.205765

I nhibitors of the DNA repair enzymes Poly(ADP-ribose)polymerase (PARP) 1/2 recently gained clinical approval by the Food and Drug Administration for the treatment of certain ovarian cancers (olaparib, rucaparib, niraparib). Simultaneously, companion diagnostics for PARP inhibitors (PARPi) are in early clinical evaluation (NCT03085147, NCT02469129, NCT03334500, NCT02637934, NCT03083288). Companion diagnostics can become powerful tools that enable patient selection, treatment planning, treatment monitoring, and diagnostic and intraoperative imaging (1). In contrast to whole-body imaging, which is predominantly based on radiolabeled PARPi, the development of fluorescent PARPi enables the interrogation of the intratumoral and

Received Nov. 21, 2017; revision accepted Feb. 12, 2018.

For correspondence or reprints contact: Susanne Kossatz, Memorial Sloan Kettering Cancer Center, 1275 York Ave., New York, NY 10065.

E-mail: Kossatzs@mskcc.org

Published online Mar. 23, 2018.

COPYRIGHT @ 2018 by the Society of Nuclear Medicine and Molecular Imaging. intracellular distribution, shedding light on their subcellular pharmacokinetics (2). Heretofore, the development of companion PARP imaging agents has required conjugation to a detectable fluorophore. The resulting modified compound is a new chemical entity, which will exhibit nonidentical properties compared with the parent PARPi. For example, the optically active PARPi derived from olaparib, PARPi-FL, maintains its cell-penetrating and specific target-binding properties but has a higher molecular weight (640 vs. $435 \mathrm{~g} / \mathrm{mol}$ ) and a slightly lower affinity (12.2 vs. $5 \mathrm{nM})(3)$.

In contrast, the clinically approved PARPi rucaparib features intrinsic fluorescence, enabling direct imaging of the actual drug without any structural modifications. Rucaparib has a molecular weight of $323 \mathrm{~g} / \mathrm{mol}$ and a PARP1 $\mathrm{K}_{\mathrm{i}}$ of $1.4 \mathrm{nM}$ (4). Rucaparib, unlike many other PARPi, has an indole chromophore embedded in its structure. This chromophore is similar to that of the intrinsically fluorescent amino acid tryptophan, which has been used to study peptides and proteins (5). In this study, we characterize the fluorescence properties of rucaparib and explore its potential to measure PARP1 expression, drug delivery, and drug-target interactions.

\section{MATERIALS AND METHODS}

\section{Spectroscopy}

Stock solutions of rucaparib in $1 \times$ Dulbecco's phosphate-buffered saline and $N$-acetyl-L-tryptophanamide (NATA; Sigma-Aldrich) in water and were made at $100 \mu \mathrm{M}$ concentrations. UV-visible spectra of rucaparib and NATA at 5 different concentrations were obtained on a Perkin-Elmer Lambda 35 UV-vis spectrometer, scanning from 200 to $1,000 \mathrm{~nm}$, in $1-\mathrm{nm}$ increments with a slit width of $1 \mathrm{~nm}$. Solutions were mixed directly in 1-cm path length quartz cuvettes, and these same cuvettes were used for steady-state and time-correlated fluorescence measurements.

Steady-state fluorescence emission and excitation spectra were obtained on a HORIBA Scientific FluoroLog-3 fluorometer. All spectra were corrected by dividing by the wavelength-dependent lamp intensity reference signal, and further baseline-corrected using the spectra of the pure solvents. For both the NATA and the rucaparib samples, emission spectra were obtained using a $290-\mathrm{nm}$ (1-nm slit width) excitation wavelength and scanning from 300 to $1,000 \mathrm{~nm}$ (5-nm slit width). The NATA excitation spectra were obtained at an emission wavelength of $400 \mathrm{~nm}$ (1-nm slit width), scanning from 200 to $390 \mathrm{~nm}$ (5-nm slit width). Rucaparib excitation spectra were obtained similarly, but monitoring emission wavelengths of 450 and $500 \mathrm{~nm}$. 
Fluorescence quantum yields were calculated using the slopes of the gradient plots of fluorescence intensity versus absorbance for the rucaparib and NATA samples, according to Equation 1.

$$
\varphi=\varphi_{\text {std }}\left(\frac{\nabla F}{\nabla F_{\text {std }}}\right)
$$

To determine the fluorescence lifetime of rucaparib, time-correlated single-photon counting (TCSPC) measurements were recorded with the FluoroHub Tau-3 module for the same fluorometer, using a diode laser source (NanoLED-405) with a pulse duration less than 200 ps. The excitation wavelength was set to $395 \mathrm{~nm}$ (slit-width $5 \mathrm{~nm}$ ), and the emission wavelength was set to 450 or $500 \mathrm{~nm}$ (both with 5-nm slit widths) to obtain 2 separate measurements for each sample concentration. The recorded decay was fit to a monoexponential function using the Decay Analysis Software (version 6.4) provided with the instrument.

\section{Cell Culture}

We used different tumor cell lines to evaluate rucaparib binding across a variety of tumor types and PARP1 expression levels. Cells were cultivated in monolayer culture at $37^{\circ} \mathrm{C}$ in a $5 \% \mathrm{CO}_{2}$ humidified atmosphere, following standard procedures. They were maintained in their respective growth medium (Roswell Park Memorial Institute medium for M21, JHU-LX22, 4T1; McCoy's for HT-29; Dulbecco modified Eagle medium for OV90; and minimum essential medium for U87), containing $10 \%$ (v/v) fetal bovine serum and $1 \%$ penicillin/ streptavidin.

\section{Time-Lapse Imaging}

HT-29 cells were grown in poly-L-lysine-coated $\mu$-slides (Ibidi). On a confocal microscope, equipped with a heated incubation chamber providing $5 \% \mathrm{CO}_{2}$, time-lapse imaging was initiated using a 405 $\mathrm{nm}$ laser for excitation and a 420- to 480-nm emission filter, with an image acquisition setting of 5-s intervals for a total of $10 \mathrm{~min}$. At $15 \mathrm{~s}$, rucaparib was added to the medium to a final concentration of $5 \mu \mathrm{M}$, and after 5 min rucaparib-containing medium was removed, fresh medium was added, and imaging was continued for another $5 \mathrm{~min}$. To determine specificity of the uptake, M21 cells were grown on polyL-lysine-coated $\mu$-slides and imaged on a SP8 confocal microscope for $60 \mathrm{~min}$. Field-of-views were set up to be recorded in adjacent wells to simultaneously observe rucaparib uptake with and without blocking with a 10-fold molar excess of olaparib. FIJI was used for image analysis.

\section{Confocal Microscopy in Different Cell Lines}

To observe rucaparib binding in different cell lines, HT-29, OV90, M21, and U87 cells were grown in 8-well chamber slides and incubated with $5 \mu \mathrm{M}$ rucaparib for $30 \mathrm{~min}$, followed by a 10 -min wash in culture medium. Cells were then fixed with $4 \%$ paraformaldehyde, nuclei were costained with the far-red fluorescent DNA dye DRAQ5, slides were mounted with Mowiol and imaged on an LSM 880 confocal microscope. To compare PARPi-FL and rucaparib uptake, cells were incubated with a mixture of both compounds at equal concentration $(5 \mu \mathrm{M})$ for $30 \mathrm{~min}$ and processed as described above. To determine differences in rucaparib uptake over time in high and low PARP1-expressing cell lines, M21 and U87 cells were incubated for different durations ( $5 \mu \mathrm{M}$ rucaparib for 5 or $40 \mathrm{~min}$ ) or at different concentrations $(0,0.5,1,5,10$, and $25 \mu \mathrm{M}$ rucaparib for $20 \mathrm{~min})$. Rucaparib incubation was followed by a 10-min washing step in fresh media in all cases. All images were obtained using the same microscope setting and were set to identical window/level settings for rucaparib using FIJI.

\section{Cryosection Imaging}

Animals studies were done in accordance with the Institutional Animal Care and Use Committee. Athymic nude mice $(n=1$ per cell line) were xenografted with $4 \mathrm{~T} 1$ or M21 cells ( 2 mio cells per animal). After 7 days, animals were intravenously injected with $200 \mathrm{nmol}$ of rucaparib and sacrificed $60 \mathrm{~min}$ later. Tumors were excised and split in 2 parts. One part was frozen in optimal-cutting-temperature (OCT) compound, and the other part was fixed in $4 \%$ paraformaldehyde before paraffin-embedding and immunohistochemical staining. We acquired $10-\mu \mathrm{m}$ cryosections from the OCT block that were imaged using a confocal microscope. Rucarib was detected in the DAPI channel.

\section{Immunohistochemistry}

Immunohistochemical detection of PARP was carried out on FFPEembedded 3- $\mu \mathrm{m}$ tumor sections using the automated Discovery XT processor (Ventana Medical Systems). After deparaffinization and antigen retrieval, anti-PARP1 antibody $(0.2 \mu \mathrm{g} / \mathrm{ml})$ was incubated for 5 $\mathrm{h}$, followed by $1 \mathrm{~h}$ of incubation with biotinylated goat anti-rabbit IgG (PK6106; Vector Labs) at a 1:200 dilution. For immunohistochemistry detection, a DAB detection kit (Ventana Medical Systems) was used according to the manufacturer's instructions. Sections were counterstained with hematoxylin and cover-slipped with Permount (Fisher Scientific).

\section{Western Blot}

PARP1 protein expression was measured with Western blot analysis. Proteins were isolated from cells, and $30 \mu \mathrm{g}$ of protein per sample were separated with sodium dodecyl sulfate polyacrylamide gel electrophores and transferred to a nitrocellulose membrane. Proteins were detected using antibodies specific for PARP1 $(1: 1,000$; sc-7150, Santa Cruz) and b-actin (1:2,000; A3854, Sigma-Aldrich) with a corresponding horseradish peroxidase-conjugated secondary antibody (1:10,000; sc-2004, Santa-Cruz). Detection was done using chemiluminescent substrate (Thermo Scientific \#34077, SuperSignal West Pico).

\section{Intracellular Half-Life of Rucaparib}

JHU-LX22 cells $(200,000$ cells/well in 6-well plates seeded $24 \mathrm{~h}$ before were incubated with $0.2 \mu \mathrm{M}$ rucaparib for $20 \mathrm{~min}$ at $37^{\circ} \mathrm{C}$ and washed for $10 \mathrm{~min}$ in medium. Then, fresh medium was added for a postincubation time of $0,1,2,4,8,12,24$, or $48 \mathrm{~h}$. Subsequently, cells were incubated with $0.2 \mu \mathrm{M}$ PARPi-FL for $20 \mathrm{~min}$ at $37^{\circ} \mathrm{C}$, followed by a 10-min wash in medium to allow for washout of unbound PARPiFL. Then, cells were trypsinized, washed with flow buffer ( $1 \%$ bovine serum albumin $[\mathrm{w} / \mathrm{v}]$ in phosphate-buffered saline), transferred to 5$\mathrm{mL}$ flow cytometry tubes through the $40-\mu \mathrm{m}$ cell strainer cap, and left on ice until flow cytometry (Fortessa II; BD Biosciences). Raw data were processed in FlowJo software to calculate rucaparib and PARPiFL uptake per cell. Cell clumps and debris were eliminated using the corresponding gates (forward and side scatter) for the unstained cell population. Rucaparib fluorescence was measured in the DAPI channel, and PARPi-FL fluorescence was imaged in the fluorescein isothiocyanate channel. Data were analyzed as mean fluorescence intensity (MFI) or normalized MFI (nMFI). For rucaparib, 100\% nMFI represents the MFI of rucaparib at $0 \mathrm{~h}$. For the indirect measurement through PARPi-FL, the highest MFI value was defined as $100 \%$ and the nMFI of every time point was subtracted from 100. Decay curves were calculated with Prism using a 2-phase decay leastsquares regression. The experiment was carried out 3 times (with technical triplicates). 


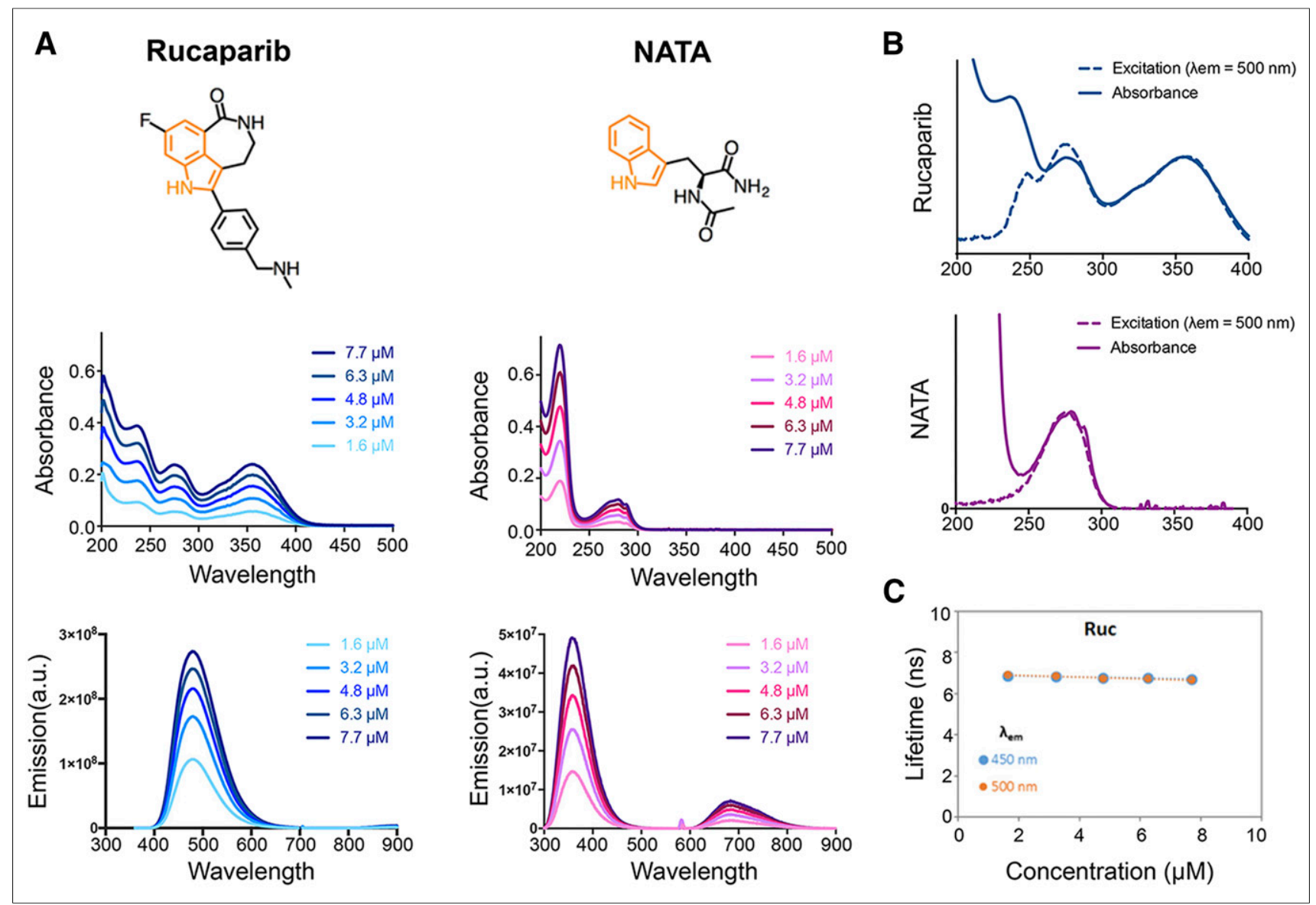

FIGURE 1. Spectroscopic characterization of rucaparib. (A) Chemical structures with highlighted indole chromophore (orange) and absorbance and emission spectra of rucaparib and NATA at 5 concentrations in water. (B) Fluorescence excitation spectra compared with absorbance (C) and fluorescence lifetime measurement of rucaparib at the emission wavelengths 450 and $500 \mathrm{~nm}$ in different concentrations in water.

\section{RESULTS}

\section{Fluorescence Spectroscopy}

We characterized the intrinsic fluorescence of rucaparib in comparison to NATA, a neutral tryptophan analog, which served as reference standard. The structures and position of the indole chromophore of both molecules are displayed in Figure 1A.

The absorbance maxima for rucaparib at 275 and $355 \mathrm{~nm}$ and for NATA at 220 and $280 \mathrm{~nm}$ are independent of concentration, suggesting that fluorophore aggregation was negligible in $\mu \mathrm{M}$ concentrations in water (Fig. 1A). The NATA emission spectra $\left(\lambda_{\mathrm{ex}}=290 \mathrm{~nm}\right)$ has a major peak around $360 \mathrm{~nm}(6)$, consistent with other indoles, whereas the rucaparib emission $\left(\lambda_{\mathrm{ex}}=290\right.$ $\mathrm{nm}$ ) peak is at $480 \mathrm{~nm}$ (Fig. 1A). The quantum yield $\varphi$ for rucaparib is 0.30 , which is significantly higher than NATA $\left(\varphi_{\text {std }}=0.13\right.$ $(7,8))$. The excitation spectra of rucaparib matched the absorbance bands at 280 and $350 \mathrm{~nm}$ (Fig. 1B).

The fluorescence lifetime for rucaparib was measured as $6.8 \mathrm{~ns}$, which is greater than the reported lifetimes of NATA and many other indole derivatives at a similar $\mathrm{pH}(6)$. We also observed a significant $\mathrm{pH}$ dependency for the photophysical properties of rucaparib (Supplemental Fig. 1; supplemental materials are available at http://jnm.snmjournals.org) that may afford other diagnostic capabilities and future studies on the interactions of rucaparib with PARP1 and other proteins.

\section{Rucaparib Live Cell Imaging}

The rucaparib signal appeared within the cell nucleoli within 30 $\mathrm{s}$ of addition to the medium, followed by the nuclei, indicating that detectable concentrations were first reached in these compartments due to binding and accumulation (Fig. 2A; Supplemental Video 1). The cytoplasmic signal was low, with the exception of a punctate extranuclear uptake, which rapidly disappeared after washing (Fig. 2A). Specificity of the accumulation was confirmed by simultaneous addition of rucaparib and a blocking dose of nonfluorescent olaparib, which strongly reduced rucaparib accumulation (Fig. 2B).

\section{Characterization of Intracellular Rucaparib Uptake}

We characterized how rucaparib fluorescence can be used to visualize PARP1 expression levels in a set of cell lines (Fig. 3). First, we determined PARP1 expression levels for all cell lines via Western blot, which were from highest to lowest HT29, M21, JHU-LX22, OV90, U87, and 4T1 (Fig. 3A). In agreement with PARP1 protein expression levels, we identified high (HT29), intermediate (OV90), and low (4T1) rucaparib uptake using confocal microscopy (Fig. 3B). We further investigated the ability of rucaparib to distinguish between different PARP1 expression levels by varying either the incubation time ( $5 \mathrm{vs} .40 \mathrm{~min}$ ) or the concentration ( 5 vs. $25 \mu \mathrm{M}$ ) in M21 and U87 cells. For both conditions, we found a stronger rucaparib signal increase in M21 cells, which express higher levels of PARP1 than U87 cells (Fig. 3C). 


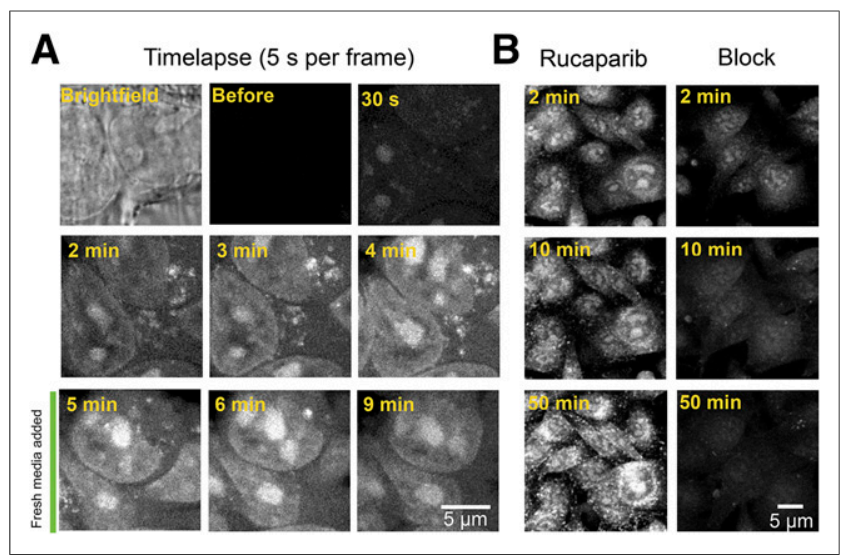

FIGURE 2. Live cell imaging of rucaparib uptake. (A) Single images from a time-lapse video of HT29 cells during incubation with $5 \mu \mathrm{M}$ rucaparib allows observation of cell penetration and nuclear accumulation. (B) Uptake of rucaparib with and without block (rucaparib mixed with a 10-fold molar excess of olaparib) in M21 cells to show specificity of rucaparib uptake.

Furthermore, we compared rucaparib uptake with the fluorescently labeled olaparib derivative, PARPi-FL, which has been previously validated to be quantitative for PARP1 expression (2). Coincubation of rucaparib and PARPi-FL led to overlapping signals of both compounds in cell nuclei, which was strong in HT29 cells (high PARP1) and weak in 4T1 cells (low PARP1) (Fig. 3D). This was also confirmed by imaging cryosections of M21 and 4T1 tumors that were excised $60 \mathrm{~min}$ after systemic injection of rucaparib. The strong rucaparib signal in M21 sections and the weak rucaparib signal in 4T1 sections were confirmed by concordant immunohistochemical PARP1 staining (Fig. 3D).

\section{On-Target Binding Time of Rucaparib}

We measured how long rucaparib resides within a cell as readout for the inhibition of DNA repair. We determined the on-target binding time by directly measuring rucaparib and confirmed the results using the established PARP imaging agent PARPi-FL as control to indirectly measure rucaparib binding. We observed a 2-phase decay of the rucaparib signal over $48 \mathrm{~h}$ and a corresponding increase of the PARPi-FL signal since it can bind only to unoccupied binding sites (Fig. 4A). We calculated the on-target half-life of rucaparib for both methods and found similar half-lives of $5.1 \mathrm{~h}$ (direct) and $6.4 \mathrm{~h}$ (indirect) (Fig. 4B), confirming the possibility of measuring drug pharmacokinetics directly through rucaparib fluorescence.

For validation of the results, we determined interactions between the 2 PARPi by measuring whether rucaparib binding was influenced by the presence of PARPi-FL and vice versa. We found that binding of rucaparib was not affected by PARPi-FL at the conditions used for determining the half-life of rucaparib. However, at longer incubation times and excess concentrations of PARPi-FL, rucaparib was released faster, which is in concurrence with the fact that both compounds compete for the same binding site. A similar effect was observed when PARPi-FL incubation was followed by rucaparib (Supplemental Fig. 2).

Taken together, these experiments show the potential to measure the intracellular dynamics and target interaction of rucaparib using its intrinsic fluorescence.

\section{DISCUSSION}

The PARPi rucaparib has recently been approved for the treatment of ovarian cancer and is in late-stage clinical trials of a variety of tumor types. In this study, we report that the intrinsic fluorescence signal of rucaparib allows the direct visual investigation of the PARP-dependent intracellular binding properties and distribution at the subcellular level. Notably, this is the clinically used drug without any structural modifications or conjugations.

The fluorescence of rucaparib arises from the core indole chromophore motif, similar to tryptophan. The increased quantum yield and lifetime are likely due to the fused 7-member heterocyclic amide, which enhances structural rigidity and thereby dampens vibrational relaxation to the ground state. Several factors contribute to the substantial redshift observed in the absorption and emission spectra relative to tryptophan. The fluorine and amide moieties inductively withdraw electron density from the aromatic ring, whereas the latter can also partially conjugate to it through resonance. Additionally, the minimum energy conformation of the phenyl substituent may have a dihedral angle close to $45^{\circ}$, similar to biphenyl, which could substantially perturb the photophysical

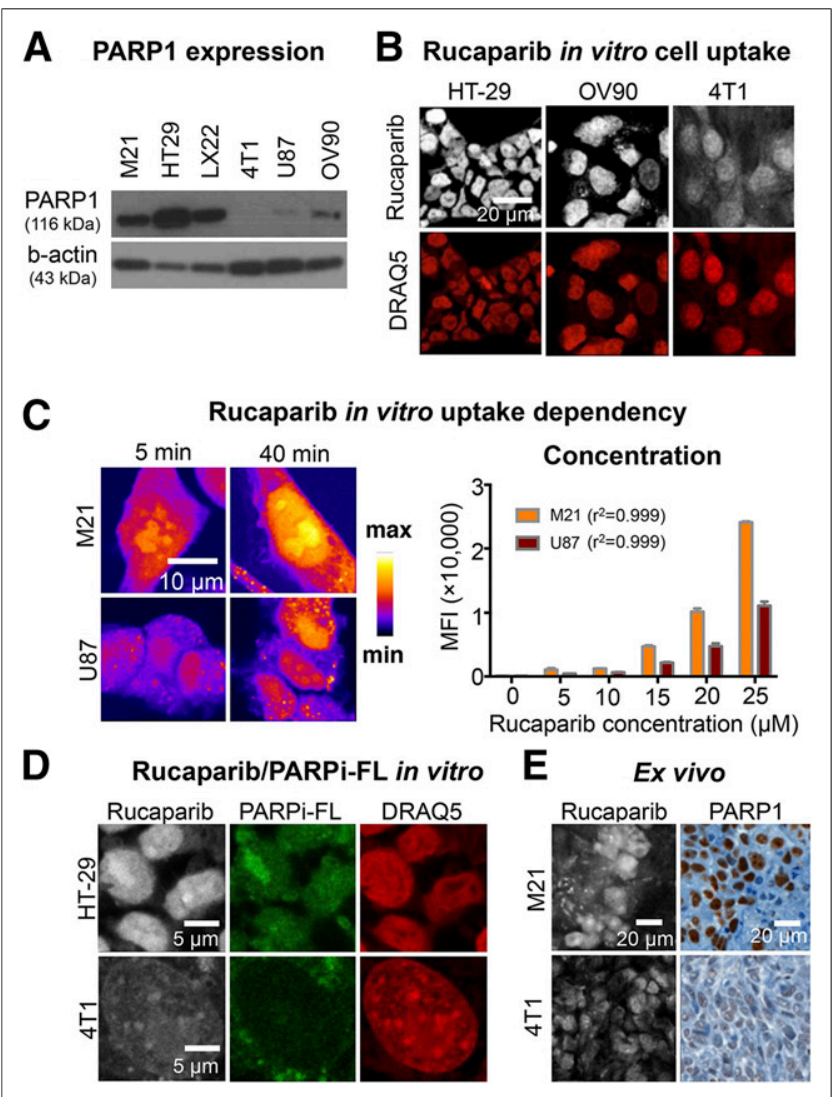

FIGURE 3. Measurement of PARP1 expression using rucaparib. (A) Western blot of PARP1 protein content of investigated cell lines using anti-PARP1 antibody and $\beta$-actin as loading control. (B) Comparison of rucaparib binding in tumor cell lines HT29, OV90, and 4T1 after a 30-min incubation with $5 \mu \mathrm{M}$ rucaparib. Nuclei were counterstained with DRAQ5, and cells were fixed before imaging. (C) Modulation of incubation time and incubation concentration in M21 (high-PARP1) and U87 (low-PARP1) cells. (D) Comparison of rucaparib and PARPi-FL uptake in HT29 and 4T1 cells after simultaneous incubation at $5 \mu \mathrm{M}$. Nuclei were counterstained with DRAQ5, and cells were fixed before imaging. Images of each channel are comparable due to identical window/level settings. (E) Rucaparib detection in cryosections of M21 or 4T1 tumor-bearing mice compared with PARP1 immunohistochemistry. Mice received intravenous injection of 200 $\mathrm{nmol}$ rucaparib and were sacrificed $60 \mathrm{~min}$ after injection. 


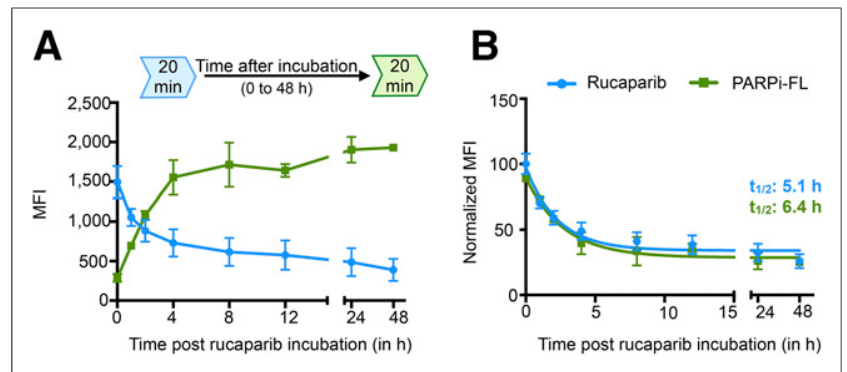

FIGURE 4. Quantification of intracellular retention of rucaparib. (A) Mean fluorescence intensities (MFI) of rucaparib and PARPi-FL over $48 \mathrm{~h}$ to observe clearance of rucaparib over time. (B) Normalized MFI to display on-target half-life of rucaparib measured directly via rucaparib or indirectly via PARPi-FL. Rucaparib and PARPi-FL were incubated at $0.2 \mu \mathrm{M}$. Decay curves were calculated with Prism using a 2-phase decay least-squares regression. Experiment was repeated 3 times with technical triplicates.

properties (9). The redshifted absorption and emission of rucaparib permit the use of conventional excitation sources $(355,405 \mathrm{~nm})$ in microscopes and flow cytometers, whereas its higher quantum yield allows for more sensitive detection of the fluorescence signal. Similar to tryptophan, rucaparib exhibits $\mathrm{pH}$-dependent fluorescent properties that can be used to further explore the nature of the binding pocket and intermolecular interactions.

We demonstrate that rucaparib uptake imaging assays clearly distinguish between cells and xenografts expressing high levels and low levels of PARP1 without the need of laborious and expensive staining procedures or labeling. Furthermore, the ability to directly measure dynamics of rucaparib uptake and clearance can have important implications in the evaluation of clinical studies involving rucaparib. Interestingly, live cell imaging revealed a pattern of extranuclear uptake of rucaparib, potentially due to secondary targetbinding effects $(10)$.

\section{CONCLUSION}

The possibility of direct rucaparib imaging offers a broad variety of clinically relevant applications, which can complement whole-body imaging of radioactively labeled PARP imaging agents $(1,11)$. This includes an in-depth investigation of off-target binding effects and establishing rucaparib as an ex vivo tool during clinical studies to monitor intracellular drug levels, for example, in liquid cancers. Furthermore, heterogeneity of drug delivery, which is a potential source for resistance to
PARPi therapy, can be investigated at a high spatial resolution using rucaparib imaging.

There are ongoing clinical trials to evaluate rucaparib, including breast cancer, ovarian cancer, fallopian tube cancer, pancreatic cancer, and prostate cancer. The identification of patients who will most likely benefit from rucaparib therapy and the optimization of treatment regimens will be of critical importance to improve outcomes for patients.

\section{DISCLOSURE}

This work was supported by National Institutes of Health grants NIH R01CA204441 (to Thomas Reiner), R21CA191679 (to Thomas Reiner), and P30 CA008748. We thank the Center for Molecular Imaging and Nanotechnology, the Tow Fellowship Program in Molecular Imaging and Nanotechnology (for support to Brandon Carney and Susanne Kossatz), the National Science Foundation Integrative Graduate Education and Research Traineeship 0965983, and CHE-161-755 (to Charles M. Drain). No other potential conflict of interest relevant to this article was reported.

\section{REFERENCES}

1. Carney B, Kossatz S, Reiner T. Molecular imaging of PARP. J Nucl Med. 2017;58:1025-1030.

2. Thurber GM, Yang KS, Reiner T, et al. Single-cell and subcellular pharmacokinetic imaging allows insight into drug action in vivo. Nat Commun. 2013;4:1504.

3. Irwin CP, Portorreal Y, Brand C, et al. PARPi-FL: a fluorescent PARP1 inhibitor for glioblastoma imaging. Neoplasia. 2014;16:432-440.

4. Rouleau M, Patel A, Hendzel MJ, Kaufmann SH, Poirier GG. PARP inhibition: PARP1 and beyond. Nat Rev Cancer. 2010;10:293-301.

5. Chen Y, Barkley MD. Toward understanding tryptophan fluorescence in proteins. Biochemistry. 1998;37:9976-9982.

6. Eftink MR, Jia J, Hu D, Ghiron CA. Fluorescence studies with tryptophan analogs: excited state interactions involving the side chain amino group. J Phys Chem. 1995;99:5713-5723.

7. Muiño PL, Callis PR. Solvent effects on the fluorescence quenching of tryptophan by amides via electron transfer: experimental and computational studies. J Phys Chem B. 2009;113:2572-2577.

8. Chen RF. Fluorescence quantum yields of tryptophan and tyrosine. Anal Lett. $1967 ; 1: 35-42$.

9. Johansson MP, Olsen J. Torsional barriers and equilibrium angle of biphenyl: reconciling theory with experiment. J Chem Theory Comput. 2008;4:1460-1471.

10. Knezevic CE, Wright G, Rix LLR, et al. Proteome-wide profiling of clinical PARP inhibitors reveals compound-specific secondary targets. Cell Chem Biol. 2016;23:1490-1503.

11. Makvandi M, Xu K, Lieberman BP, et al. A radiotracer strategy to quantify PARP-1 expression in vivo provides a biomarker that can enable patient selection for PARP inhibitor therapy. Cancer Res. 2016;76:4516-4524. 